\title{
Voids' layer structures in silicon irradiated with high doses of high-energy helium ions
}

\author{
M.I. Starchyk, L.S. Marchenko, M.B. Pinkovska, G.G. Shmatko, V.I. Varnina \\ Institute for Nuclear Research, National Academy of Science of Ukraine, \\ 47, prospect Nauky 03028 Kyiv, Ukraine; phone 38(044) 525 3749, e-mail: myrglory@yahoo.com
}

\begin{abstract}
Structural and optical properties of single crystal silicon irradiated with 27.2 MeV helium ions by using fluences $\Phi \geq 10^{16} \mathrm{ion} / \mathrm{cm}^{2}$ were studied at various beam currents. It was found that at currents 0.25 to $0.45 \mu \mathrm{A}$, heavily damaged layers containing voids were formed in ion path in $\mathrm{Si}$ and behind it. The number of layers in the ion path region depends on the beam intensity. With increasing the beam current up to $\sim 1 \mu \mathrm{A}$, the layer structures consisting of voids were observed only in the ion path. As helium is poorly soluble in $\mathrm{Si}$, during implantation it collects in the gas-filled vacancy complexes. We consider that, like to the case of $\mathrm{keV}$-ion implantation at fluences of $\Phi \geq 10^{16} \mathrm{ion} / \mathrm{cm}^{2}$, an amorphous layer is created in the ion stopping region at annealing. Moving by recrystallization fronts on both sides of the amorphous layer, vacancy clusters are collected inside, coalesce and form voids. It is a combination of high energy and high fluence helium implantation that can form layered structure with voids in silicon, as observed by us. At present, there is no strict explanation of the mechanism of voids' ordering (forming of superlattice of them). Especially, it concerns the void layer formation beyond helium ion path. The concept of mobile solitons is used. Formation of the "lattice" from the voids leads to swelling of the material. Further researches are necessary to understand these processes and control them.
\end{abstract}

Keywords: silicon, helium implantation, high defect concentration, layer structures, voids.

Manuscript received 06.04.15; revised version received 16.06.15; accepted for publication 03.09.15; published online 30.09.15.

\section{Introduction}

Theoretical and experimental data received in the field of radiation solid state physics indicate the possibility of self-organization in the ensemble of radiation defects, especially under ion implantation [1-3]. An important factor for the radiation modification of material properties is information on the distribution of ions and structural defects in the irradiated material, their size, thermal stability and so on. The results are of fundamental importance for better understanding the nature of interaction of radiation with solids at high concentration of defects.
Interest in the behaviour of helium implanted in silicon is related with the fact of voids' formation at high fluences of irradiation $[4,5]$. Helium is poorly soluble in $\mathrm{Si}$ and during irradiation collects in the gas-filled vacancy complexes. When silicon is heated at temperatures above $T \geq 400{ }^{\circ} \mathrm{C}$, these complexes grow and helium are released, leaving voids. Surface of voids remains clean, making them places of possible gettering of impurities from the surrounding matrix, as well as centres of strain relaxation in the silicon lattice.

We present the results of studying the structural and optical properties of monocrystalline silicon irradiated with helium ions possessing the energy close 
to $27.2 \mathrm{MeV}$ at fluences $\geq 5 \cdot 10^{16} \mathrm{ion} / \mathrm{cm}^{2}$ by varying the beam current. The goal of the work is to obtain information about the nature and parameters of the defect structure and optical properties of irradiated silicon.

\section{Experimental}

Silicon samples were made using the Czochralsky method $(\langle 111\rangle, p$-type, $\rho=10 \mathrm{Ohm} \cdot \mathrm{cm})$ and irradiated with helium ions with an energy of $27.2 \mathrm{MeV}$ at fluences of $10^{16}$ to $10^{17} \mathrm{ion} / \mathrm{cm}^{2}$ by varying the beam current from 0.25 to $1.0 \mu \mathrm{A}$ in the cyclotron U-120 of Institute for Nuclear Research, NAS of Ukraine. During irradiation, the samples were cooled with running water. The projection length of the path of ions with a given energy is about $360 \mu \mathrm{m}$. The irradiated samples were cut along the direction of irradiation, allowing to study the properties of silicon in the region of ions' path, braking and behind stopping parts.

\section{Results}

X-ray topography of irradiated silicon sample, after polishing and chemical etching, displayed significant disruption of the structure in the region of ion stopping at the depth approximately $360 \mu \mathrm{m}$ (Fig. 1).

In the micrographs of silicon surface obtained by scanning electron microscopy (Fig. 2) after irradiation of two different samples $(\mathrm{a}, \mathrm{b})$ with the fluence $10^{17} \mathrm{~cm}^{-2}$ at a beam current of $\sim 0.25 \ldots 0.45 \mu \mathrm{A}$, the lines of strains are visible. Distances of lines from the irradiated surface are shown in Table. Lines (III-IV) are associated with the end of the path of helium ions. Other lines are shown within the path and behind the stopping part of the sample (long-range effect). When the beam current increases up to $1 \mu \mathrm{A}$, lines of the strain appeared only in the path region of ions. Long-range effect was not observed. The number of strain lines in the path region of the sample was determined likely by the temperature distribution over the irradiated surface, declining from the centre (c) to the edge $(a, b)$ of the sample, where the maximum cooling took place (Fig. 3).

Table. Distances of “defect walls" from the sample surface.

\begin{tabular}{|c|c|c|}
\hline \multirow{2}{*}{$\begin{array}{c}\text { Number } \\
\text { of the "wall" }\end{array}$} & \multicolumn{2}{|c|}{$\begin{array}{c}\text { Distances of "defect walls" } \\
\text { from the sample surface, } \mu \mathrm{m}\end{array}$} \\
\cline { 2 - 3 } & Metallography & $\begin{array}{c}\text { Scanning electron } \\
\text { microscopy }\end{array}$ \\
\hline I & 132 & 150 \\
\hline II & 242 & 282 \\
\hline III & 341 & 362 \\
\hline IV & 380 & 385 \\
\hline V & 423 & - \\
\hline VI & 627 & 636 \\
\hline VII & 720 & 674 \\
\hline VIII & 764 & - \\
\hline
\end{tabular}

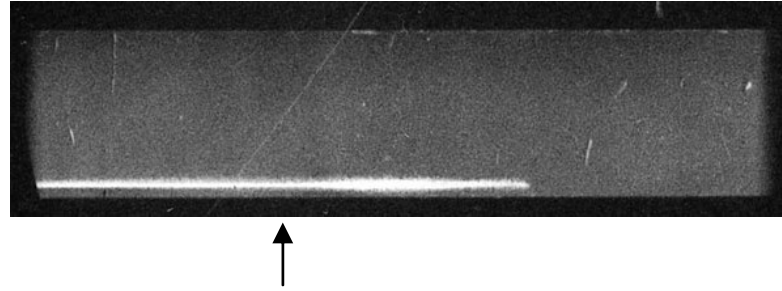

Fig. 1. X-ray topogram of the irradiated silicon sample $(18 \times 4 \mathrm{~mm})$.
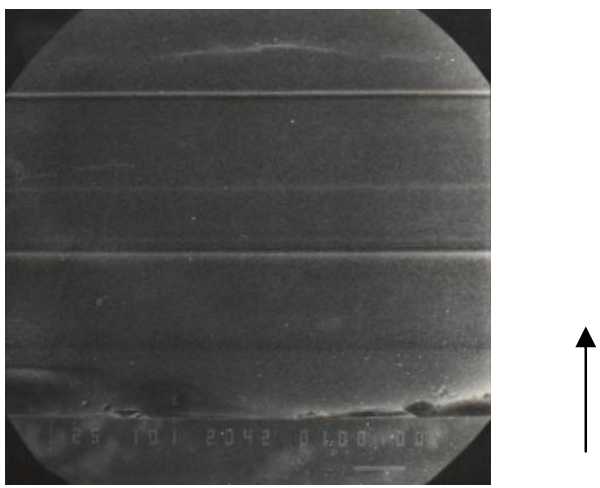

$\mathrm{a}$

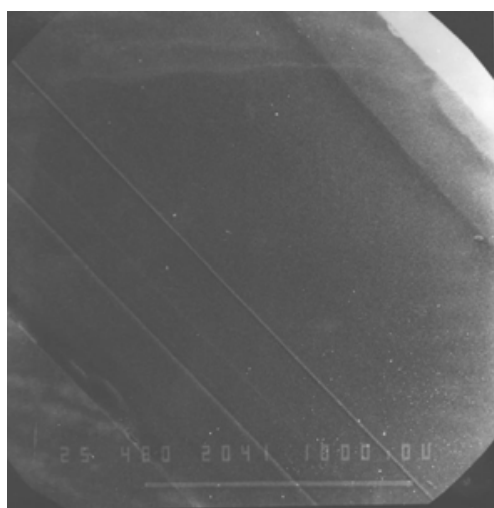

b

Fig. 2. The micrographs of silicon surface obtained by scanning electron microscopy after irradiation of two different samples $(\mathrm{a}, \mathrm{b})$ with the fluence $10^{17} \mathrm{ion} / \mathrm{cm}^{2}$ at the beam current $0.3 \mu \mathrm{A}$. Arrows indicate the direction of irradiation.

Microprofilogram of the sample surface (Fig. 4) shows the swelling of silicon in the region of the helium ions' path. The difference in the profile of the irradiated (a) and unexposed (b) parts of the sample is about $8 \mu \mathrm{m}$. The dot-dashed line indicates the position of straggling (stopping) of ions.

Images of the surfaces of the initial and irradiated Si samples $\left(10^{17} \mathrm{ion} / \mathrm{cm}^{2}\right)$ obtained using the atomic force microscope (Fig. 5) confirm the swelling of $\mathrm{Si}$ in the path region of ions: roughness of the irradiated surface greatly increased in comparison with the initial one [7]. 


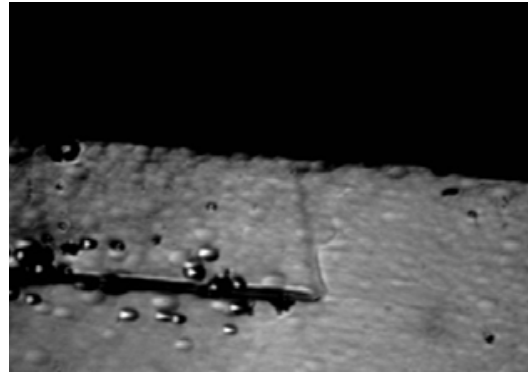

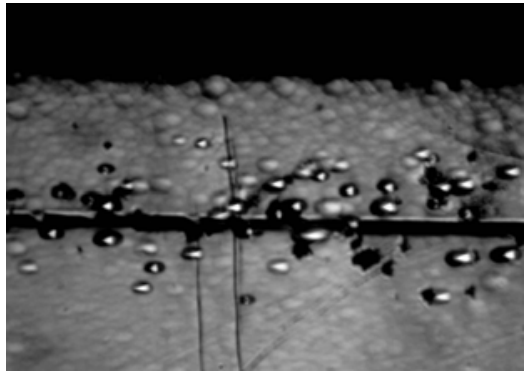

$\mathrm{b}$

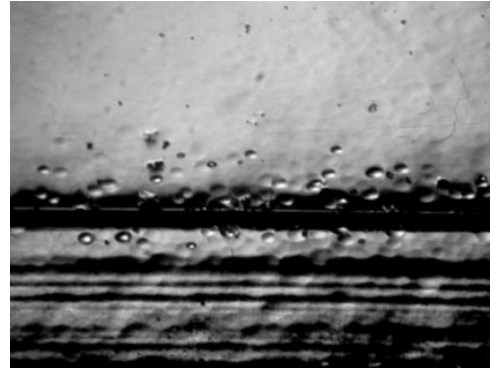

Fig. 3. Picture of the selective etching of Si irradiated with the fluence $10^{17} \mathrm{ion} / \mathrm{cm}^{2}$ at the beam current $1.0 \mu \mathrm{A}(a, b-$ at the edge, $c$ - in the centre of irradiated region of the sample).

Results of metallographic study for the Si sample irradiated up to the dose $10^{17} \mathrm{~cm}^{-2}$ at the ion beam current close to $1 \mu \mathrm{A}$ after selective etching are shown in Fig. 6. It is seen that the strain lines in the path region of the sample are the layers consisting of voids with different shapes and sizes. Voids were observed in the layers (a) and isolated from each other (b). The number of layers depends on the intensity of the ion beam and, hence, the heating temperature of the sample during irradiation. Fig. 3c shows two well-observed etched layers: upper one - the width 30 to $45 \mu \mathrm{m}$ (borderline of ion stopping); lower one - the width 10 to $15 \mu \mathrm{m}$ located closer to the irradiated surface. Besides, voids near the stacking faults and dislocations were observed in the vicinity of the layers. Strain lines at a distance greater than the mean free path of ions (long-range effect) were observed at the beam current 0.25 to $0.45 \mu \mathrm{A}$ and disappeared as the current increases to $\sim 1 \mu \mathrm{A}$. IR absorption spectra of silicon studied using the Fourier spectrometer FIS-113V at room temperature with a spectral resolution $1.0 \mathrm{~cm}^{-1}$ are shown in Fig. 7. In the differential absorption spectra of silicon irradiated with the ion fluences of $5 \cdot 10^{16}$ and $1 \cdot 10^{17} \mathrm{ion} / \mathrm{cm}^{2}$ (curves 2,3) an additional absorption associated with the irradiation was not observed within the spectral range $400 \ldots .5000 \mathrm{~cm}^{-1}$.

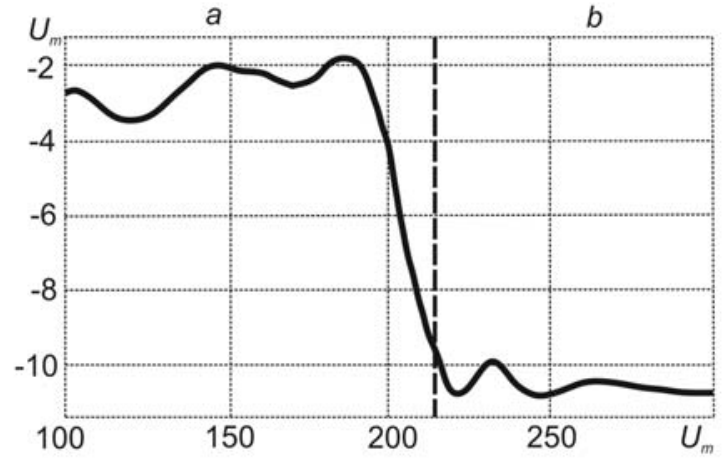

Fig. 4. Microprofilogram of Si suface after irradiation $(a-$ ion path region, $b$-behind it). The dashed line indicates struggling.

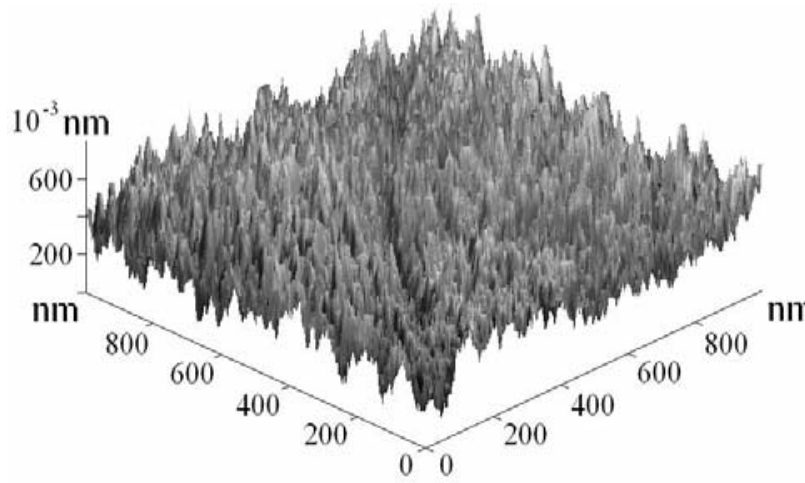

a

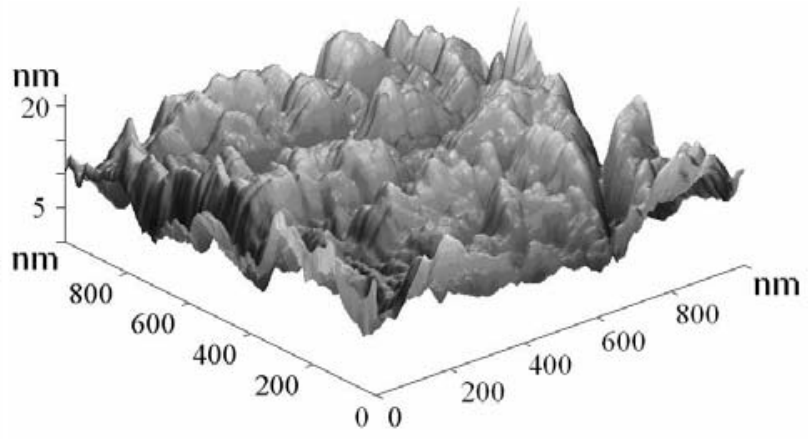

b

Fig. 5. AFM image of the Si surface before (a) and after irradiation $(b)$. 

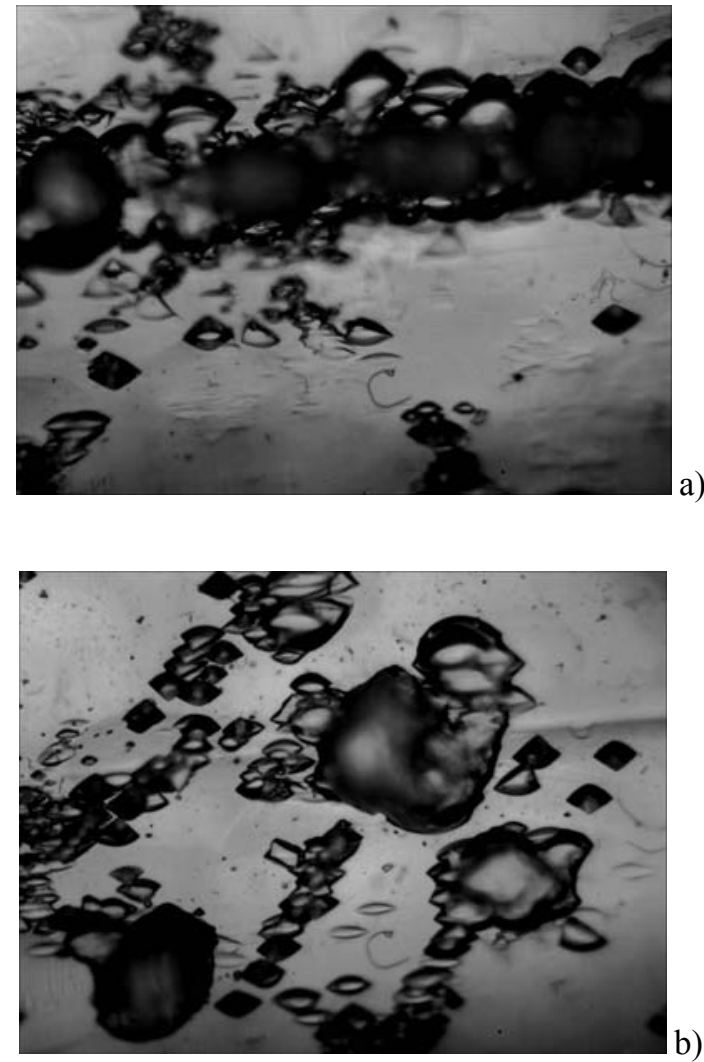

Fig. 6. Voids and defects in the ion path region of irradiated Si, which were revealed using the selective etching.

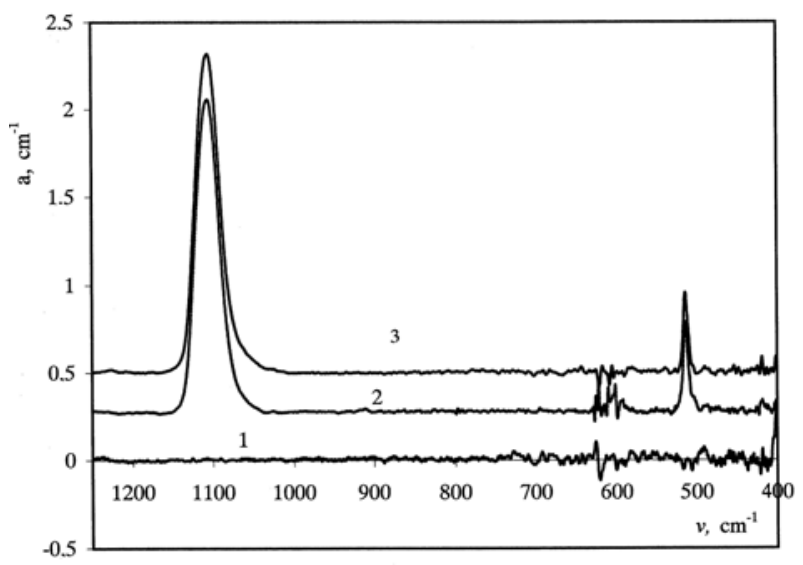

Fig. 7. Differential absorption spectra of Si irradiated with helium ions: $1(\mathrm{Si}-5)-\Phi=5 \cdot 10^{16} \mathrm{ion} / \mathrm{cm}^{2} ; 2(\mathrm{Si}-6)-\Phi=$ $1 \cdot 10^{17} \mathrm{ion} / \mathrm{cm}^{2} ; 3(\mathrm{Si}-7)-\Phi=0$.

\section{Discussion}

It is known [5] that, in silicon irradiated at room temperature with ions possessing the energy of tens $\mathrm{keV}$ and the fluence of $\Phi \geq 10^{16} \mathrm{ion} / \mathrm{cm}^{2}$, an amorphous layer is created in the ion stopping region. The centre of the amorphous region lies in the range with the highest number of displaced atoms. The thickness of the amorphous layer increases with increasing the irradiation fluence. Amorphization can be avoided while increasing the irradiation temperature up to $250{ }^{\circ} \mathrm{C}$ and above.

Clustering of vacancies in the case of $\mathrm{He}$ implantation manifested itself in the form of formation of gas-vacancies complexes, and excess interstitial atoms form extended defects (stacking faults, rod-like defects and ribbon-like defects) [8]. Increasing the irradiation temperature promotes releasing of gas from the vacancy complexes, and then voids remain. Formation of the vacancy complexes is possible with a high concentration of vacancies, which is determined by the concentration of disintegrated Frenkel pairs. When in our experiment we used helium ions with $\mathrm{MeV}$-energies instead of $\mathrm{keV}$, these conditions are performed better.

It is believed that the ion fluence $\Phi=10^{16} \mathrm{ion} / \mathrm{cm}^{2}$ is the threshold fluence to form gas-vacancy complexes in silicon. A more accurate value of the fluence is determined by temperature of implantation, energy and mass of ions. Upon annealing of irradiated silicon at $800{ }^{\circ} \mathrm{C}$, the restore of disintegrated amorphous structure occurs through epitaxial recrystallization on both sides of the amorphous layer [9]. The process begins at the boundary between the amorphous layer and Si matrix. Recrystallization promotes acceleration of the gas segregation, the restoring of the defect structure (amorphous layer) and formation of a polycrystalline layer.

In addition, in the case of large ion fluence, formation of periodic structures was observed in irradiated materials $[10,11]$.

At the beam current $\sim 1 \mu \mathrm{A}$ and fluence $10^{17} \mathrm{ion} / \mathrm{cm}^{2}$, in path (for helium ions) region of $\mathrm{Si}$ we observe two heavily damaged layers consisting of voids (Fig. 3c): upper one - in the region of ion stopping at a depth of $\sim 360 \mu \mathrm{m}$; lower one - closer to the irradiated surface. Behind the stopping part of silicon such defects were not detected.

When reducing the beam current to $0.25 \ldots 0.45 \mu \mathrm{A}$, the strain lines are observed in the ion path region of silicon and beyond. It shows the possibility to obtain different layer picture of the defect distribution by changing the current of the ion beam (irradiation intensity).

It is assumed [4] that during the annealing of silicon irradiated with helium ions of $\mathrm{keV}$-energy, helium-filled vacancy complexes are moving in the direction of the irradiated surface. After reaching it, helium goes of them, leaving large empty "pockets" of voids near surface. The authors of [5] explain this movement by the effect of restructuring, which is caused by the movement of the recrystallization front through the amorphous material.

Moving by the recrystallization fronts on both sides of the amorphous layer, vacancy clusters are collected inside, coalesce and form voids. It is a combination of a high energy and a high fluence implantation of helium that can form multi-layered structure of voids in silicon,

\section{(C) 2015, V. Lashkaryov Institute of Semiconductor Physics, National Academy of Sciences of Ukraine}


which is observed by us. Upon annealing of silicon irradiated with helium ions of the $\mathrm{keV}$ energy at $\sim 800{ }^{\circ} \mathrm{C}$, the amorphous region at the end of the ion path recrystallizes forming a polycrystalline region with stacking faults and dislocations [4]. For this reason, to avoid amorphization step implantation is carried out at elevated temperatures. High-energy ion irradiation provides such defect structuring, i.e., formation of enriched or depleted defect regions by varying the beam current (irradiation intensity).

The mechanisms of amorphization and recrystallization under ion irradiation of silicon are still under discussion [5]. Because of the lack of reliable data on the nature and parameters of the altered layers, it is impossible to determine the precise threshold fluence of irradiation, period and size of these superstructures.

In order to explain the observed by us the spread of ordered structures behind the stopping for helium ions region of silicon in the case of the current $0.25 \ldots 0.45 \mu \mathrm{A}$ and fluence $\Phi \geq 10^{16} \mathrm{ion} / \mathrm{cm}^{2}$, the concept of mobile solitons is used nowadays [8]. Solitons originate in the planes of cascades of atoms displacements and propagating along close-packed directions in the crystal: they are thermostable and introduced by the focusing collisions of atoms. Defects that form these structures are likely to be interstitial atoms.

\section{Conclusion}

Irradiation of monocrystalline silicon with helium ions possessing the energy $27.2 \mathrm{MeV}$ and fluence $\Phi \geq$ $10^{16} \mathrm{ion} / \mathrm{cm}^{2}$ leads to formation of linear structures within the region of ion path as well as behind them. The number of structures depends on the intensity of irradiation (beam current). At currents $\sim 0.25 \ldots 0.45 \mu \mathrm{A}$, linear structures are observed in ion path of $\mathrm{Si}$ and behind it. With increasing beam current to $\sim 1 \mu \mathrm{A}$, linear structures consisting of voids were observed only in the path region of ions.

At present, there is no other explanation of the mechanism of pores' ordering (forming of superlattice of them). Especially, it concerns the "shrinkage" or compression of pores beyond their limits. It is assumed that, when pores are along the path of propagation of solitons, the conditions should create of ordering up to creation of the "lattice" of them; for all other directions there are compression and "sweeping".

Formation of the "lattice" from the pores leads to swelling of material. Further researches are necessary to understand these processes and to control them.

\section{Acknowledgement}

The authors are grateful to Corresponding Member of NASU Vladimir Sugakov for a fruitful discussion of the results and help. The authors are also grateful to referee for careful reading the manuscript and discussion.

\section{References}

1. P.A. Selishchev, Self-organisation in Radiation Physics. Publ. "Aspect-Poligraph”, Kyiv, 2004 (in Russian).

2. D.I. Tetelbaum, V.Ya. Bayankin, Long-range Effect // Priroda, 4, p. 9-17 (2005), in Russian.

3. V.I. Sugakov, Peculiarities of formation of implanted atom density distribution beyond ion range // Yadernaia Fizika i Energetika, 10(4), p. 395-402 (2009), in Russian.

4. M. Vishniakov, S.E. Donnelly, G. Carter, The influence of impurities on the growth of $\mathrm{He}$ induced cavities in silicon // J. Appl. Phys. 94(1), p. 238-244 (2003).

5. E. Oliviero, S. Peripolli, L. Amararal et al., Damage accumulation in neon implanted silicon // Appl. Phys. 100, 043505 (2006).

6. A.A. Groza, P.G. Litovchenko, M.I. Starchyk, Radiation Effects in IR-absorption and Structure of Silicon. Naukova dumka, Kyiv, 2006 (in Ukrainian).

7. M.L. Dmytruk, O.S. Kondratenko, M.B. Pinkovska et al., Optical and sensitive properties of nanostructured silicon irradiated with high-energy particles (protons, $\alpha$-particles, and heavy ions) // Ukr. J. Phys. 55(7), p. 808-816 (2010).

8. M.F. Beufort, S.E. Donnelly, S. Rousselet, M.I. David, J.F. Barbot, Extented type defects created by high temperature implantation into $\mathrm{Si} / /$ Nucl. Inst. and Meth. in Phys. Res. B, 242, p. 565567 (2006).

9. J. Nord, K. Nordlund, J. Keinonen, Amortization mechanism and defect structure in ion-beam amorphized Si, Ge, GaAs // Phys. Rev. B, 65, 165329 (2002).

10. A.M. Myasnikov, V.I. Obodnikov, V.G. Seryapin et al., Formation of quasi-periodic boron distribution in silicon initiated by ion implantation // Semiconductors, 31(3), p. 279-282 (1997).

11. V.I. Dubinko, A.C. Gudla, S.E. Donnelly, Radiation-induced formation, annealing and ordering of voids in crystals: Theory and experiment // Nucl. Inst. and Meth. in Phys. Res. B, 269, p. 1634-1639 (2011). 